\title{
Two Algorithms for Additive and Fair Division of Mixed Manna
}

\author{
Martin Aleksandrov and Toby Walsh \\ Technical University Berlin, Germany \\ \{martin.aleksandrov, toby.walsh\}@tu-berlin.de
}

\begin{abstract}
We consider a fair division model in which agents have positive, zero and negative utilities for items. For this model, we analyse one existing fairness property - EFX - and three new and related properties - $\mathrm{EFX}_{0}, \mathrm{EFX}^{3}$ and $\mathrm{EF}^{3}{ }^{3}$ - in combination with Pareto-optimality. With general utilities, we give a modified version of an existing algorithm for computing an $\mathrm{EF}^{3}$ allocation. With $-\alpha / 0 / \alpha$ utilities, this algorithm returns an $\mathrm{EFX}^{3}$ and $\mathrm{PO}$ allocation. With absolute identical utilities, we give a new algorithm for an EFX and PO allocation. With $-\alpha / 0 / \beta$ utilities, this algorithm also returns such an allocation. We report some new impossibility results as well.
\end{abstract}

Keywords: Additive Fair division · Envy-freeness · Pareto-optimality

\section{Introduction}

Fair division of indivisible items lies on the intersection of fields such as social choice, computer science and algorithmic economics [16. Though a large body of work is devoted to the case when the items are goods (e.g. [12, 20, 23, 27]), there is a rapidly growing interest in the case of mixed manna (e.g. [5, 14, 26]). In a mixed manna, each item can be classified as mixed (i.e. some agents strictly like it and other agents strictly dislike it), good (i.e. all agents weakly like it and some agents strictly like it), bad (i.e. all agents weakly dislike it and some agents strictly dislike it) or dummy (i.e. all agents are indifferent to it).

An active line of fair division research currently focuses on approximations of envy-freeness (i.e. no agent envies another one) [19]. For example, Aziz et al. [4] proposed two such approximations for mixed manna: EF1 and EFX. EF1 requires that an agent's envy for another agent's bundle is eliminated by removing some item from these agents' bundles. EFX strengthens EF1 to any non-zero valued item in these bundles, increasing the agent's utility or decreasing the other agent's utility. However, they study only EF1 and identify improving our understanding of EFX as an important open problem for mixed manna:

"Our work paves the way for detailed examination of allocation of goods/ chores, and opens up an interesting line of research, with many problems left open to explore. In particular, there are further fairness concepts that could be studied from both existence and complexity issues, most notably envy-freeness up to the least valued item (EFX) [15]." 
We make in this paper a step forward in this direction. In particular, we study not only EFX but also new properties, all stronger than EF1. For example, one such property is envy-freeness by parts up to some item: EF $1^{3}$. This ensures EF1 independently for the set of all items, the set of goods and the set of bads (i.e. the different parts). Another such property is envy-freeness by parts up to any item: $\mathrm{EFX}^{3}$. This requires EFX for each of the different parts of the set of items. Yet a third such property is $\mathrm{EFX}_{0}$. This one extends the existing envy-freeness up to any (possibly zero valued) good from [25] to any (possibly zero valued) bad by relaxing the non-zero marginal requirements in the definition of EFX. We will shortly observe the following relations between these properties.

$$
\mathrm{EFX}_{0} \Rightarrow \mathrm{EFX} \quad \mathrm{EFX}^{3} \Rightarrow \mathrm{EFX} \quad \mathrm{EF}^{3} \Rightarrow \mathrm{EF} 1 \quad \mathrm{EFX}^{3} \Rightarrow \mathrm{EF}^{3}
$$

We analyse these properties in isolation and also in combination with an efficiency criterion such as Pareto-optimality (PO). PO ensures that we cannot make an agent happier without making another one unhappier. More precisely, we ask in our work whether combinations of these properties can be guaranteed, and also how to do this when it is possible. Our analysis covers three common domains for additive (i.e. an agent's utility for a set of items is a sum of their utilities for the items in the set) utility functions: general (i.e. each utility is realvalued), absolute identical (i.e. for each item, the agents' utilities have identical magnitudes but may have different signs) as well as ternary (i.e. each utility is $-\alpha, 0$ or $\beta$ for some $\alpha, \beta \in \mathbb{R}_{>0}$ ).

Each of these domains can be observed in practice. For instance, if a machine can perform a certain task faster than some pre-specified amount of time, then its utility for the task is positive and, otherwise, it is negative. Thus, multiple machines can have mixed utilities for tasks. Further, consider a market where items have prices and agents sell or buy items. In this context, the agent's utilities for an item have identical magnitudes but different signs. Finally, a special case of ternary utilities is when each agent have utility $-1,0$, or 1 for every item. This is practical because we need simply to elicit whether agents like, dislike or are indifferent to each item. A real-world setting with such utilities is the food bank problem studied in [1].

We give some related work, formal preliminaries and motivation in Sections 2, 3 and 4 respectively. In Section 5 , we give a polynomial-time algorithm (i.e. Algorithm 1) for computing an $\mathrm{EF}^{3}$ allocation with general utilities. We also prove that an $\mathrm{EFX}^{3}$ allocation, or an $\mathrm{EFX}_{0}$ allocation might not exist even with ternary identical utilities. In Section 6, we give a polynomial-time algorithm (i.e. Algorithm 2 ) for computing an EFX and PO allocation with absolute identical utilities, and show that Algorithm 1 returns an EF $1^{3}$ and $\mathrm{PO}$ allocation. In Section 7, we show that Algorithm 1 returns an EF1 $1^{3}$ and $\mathrm{PO}$ allocation with ternary utilities, whereas Algorithm 2 returns an EFX and PO allocation. Finally, we give a summary in Section 8 . 


\section{Related work}

For indivisible goods, EF1 was defined by Budish [13. Caragiannis et al. 15] proposed EFX. It remains an open question of whether EFX allocations exist in problems with general utilities. Recently, Amanatidis et al. 2] proved that EFX allocations exist in 2-value (i.e. each utility takes one of two values) problems. In contrast, we show that EFX and PO allocations exist in problems with ternary (i.e. $-\alpha / 0 / \beta$ ) utilities, which are special cases of 3 -value problems. Barman, Murthy and Vaish [7] presented a pseudo-polynomial time algorithm for EF1 and PO allocations. Barman et al. [8] gave an algorithm for EFX and PO allocations in problems with identical utilities. Plaut and Roughgarden [25] proved that the leximin solution from [18] is also EFX and PO in this domain. Although this solution maximizes the minimum agent's utility (i.e. the egalitarian welfare), it is intractable to find in general [17. In our work, we give a polynomial-time algorithm for EFX and PO allocations in problems with absolute identical utilities, and show that this welfare and $\mathrm{EFX}^{3}$ are incompatible.

For mixed manna, Aziz et al. 44 proposed EF1 and EFX. They gave the double round-robin algorithm that returns EF1 allocations. Unfortunately, these are not guaranteed to satisfy PO. They also gave a polynomial-time algorithm that returns allocations which are EF1 and PO in the case of 2 agents. Aziz and Rey [6] gave a "ternary flow" algorithm for leximin, EFX and PO allocations with $-\alpha / 0 / \alpha$ utilities. With $-\alpha / 0 / \beta$ utilities, we discuss that these might sadly violate $\mathrm{EFX}^{3}$ even when $\alpha=1, \beta=1$, or EFX when $\alpha=2, \beta=1$. By comparison, we give a modified version of the double round-robin algorithm that returns $\mathrm{EF} 1^{3}$ allocations in problems with general utilities, $\mathrm{EF} 1^{3}$ and $\mathrm{PO}$ allocations in problems with absolute identical utilities and $\mathrm{EFX}^{3}$ and $\mathrm{PO}$ allocations in problems with $-\alpha / 0 / \alpha$ utilities. Other works of divisible manna are [9, 10, 11], and approximations of envy-freeness for indivisible goods are [3, 15, 22. In contrast, we study some new approximations and the case of indivisible manna.

\section{Formal preliminaries}

We consider a set $[n]=\{1, \ldots, n\}$ of $n \in \mathbb{N}_{\geq 2}$ agents and a set $[m]=\{1, \ldots, m\}$ of $m \in \mathbb{N}_{\geq 1}$ indivisible items. We assume that each agent $a \in[n]$ have some utility function $u_{a}: 2^{[m]} \rightarrow \mathbb{R}$. Thus, they assign some utility $u_{a}(M)$ to each bundle $M \subseteq[m]$. We write $u_{a}(o)$ for $u_{a}(\{o\})$. We say that $u_{a}$ is additive if, for each $M \subseteq[m], u_{a}(M)=\sum_{o \in M} u_{a}(o)$. We also write $u(M)$ if, for each other agent $b \in[n], u_{a}(M)=u_{b}(M)$.

With additive utility functions, the set of items $[\mathrm{m}]$ can be partitioned into mixed items, goods, bads and dummies. Respectively, we write $[m]^{ \pm}=\{o \in$ $\left.[m] \mid \exists a \in[n]: u_{a}(o)>0, \exists b \in[n]: u_{b}(o)<0\right\},[m]^{+}=\{o \in[m] \mid \forall a \in[n]:$ $\left.u_{a}(o) \geq 0, \exists b \in[n]: u_{b}(o)>0\right\},[m]^{-}=\left\{o \in[m] \mid \forall a \in[n]: u_{a}(o) \leq 0, \exists b \in[n]:\right.$ $\left.u_{b}(o)<0\right\}$ and $[m]^{0}=\left\{o \in[m] \mid \forall a \in[n]: u_{a}(o)=0\right\}$ for the sets of these items. We refer to an item $o$ from $[m]^{+}$as a pure good if $\forall a \in[n]: u_{a}(o)>0$. Also, we refer to an item $o$ from $[m]^{-}$as a pure bad if $\forall a \in[n]: u_{a}(o)<0$. 
We say that agents have general additive utilities if, for each $a \in[n]$ and each $o \in[m], u_{a}(o)$ could be any number from $\mathbb{R}$. Further, we say that they have absolute identical additive utilities if, for each $o \in[m],\left|u_{a}(o)\right|=\left|u_{b}(o)\right|$ where $a, b \in[n]$, or identical additive utilities if, for each $o \in[m], u_{a}(o)=u_{b}(o)$ where $a, b \in[n]$. Finally, we say that agents have ternary additive utilities if, for each $a \in[n]$ and each $o \in[m], u_{a}(o) \in\{-\alpha, 0, \beta\}$ for some $\alpha, \beta \in \mathbb{R}_{>0}$.

An (complete) allocation $A=\left(A_{1}, \ldots, A_{n}\right)$ is such that (1) $A_{a}$ is the set of items allocated to agent $a \in[n],(2) \bigcup_{a \in[n]} A_{a}=[m]$ and (3) $A_{a} \cap A_{b}=\emptyset$ for each $a, b \in[n]$ with $a \neq b$. We consider several properties for allocations.

Envy-freeness up to one item Envy-freeness up to one item requires that an agent's envy for another's bundle is eliminated by removing an item from the bundles of these agents. Two notions for our model that are based on this idea are EF1 and EFX [4.

Definition 1 (EF1) An allocation $A$ is envy-free up to some item if, for each $a, b \in[n], u_{a}\left(A_{a}\right) \geq u_{a}\left(A_{b}\right)$ or $\exists o \in A_{a} \cup A_{b}$ such that $u_{a}\left(A_{a} \backslash\{o\}\right) \geq u_{a}\left(A_{b} \backslash\{o\}\right)$.

Definition 2 (EFX) An allocation $A$ is envy-free up to any non-zero valued item if, for each $a, b \in[n]$, (1) $\forall o \in A_{a}$ such that $u_{a}\left(A_{a}\right)<u_{a}\left(A_{a} \backslash\{o\}\right)$ : $u_{a}\left(A_{a} \backslash\{o\}\right) \geq u_{a}\left(A_{b}\right)$ and (2) $\forall o \in A_{b}$ such that $u_{a}\left(A_{b}\right)>u_{a}\left(A_{b} \backslash\{o\}\right)$ : $u_{a}\left(A_{a}\right) \geq u_{a}\left(A_{b} \backslash\{o\}\right)$.

Plaut and Roughgarden [25] considered a variant of EFX for goods where, for any given pair of agents, the removed item may be valued with zero utility by the envy agent. Kyropoulou et al. 21] referred to this one as $\mathrm{EFX}_{0}$. We adapt this property to our model.

Definition $3\left(\mathrm{EFX}_{0}\right)$ An allocation $A$ is envy-free up to any item if, for each $a, b \in[n],(1) \forall o \in A_{a}$ such that $u_{a}\left(A_{a}\right) \leq u_{a}\left(A_{a} \backslash\{o\}\right): u_{a}\left(A_{a} \backslash\{o\}\right) \geq u_{a}\left(A_{b}\right)$ and (2) $\forall o \in A_{b}$ such that $u_{a}\left(A_{b}\right) \geq u_{a}\left(A_{b} \backslash\{o\}\right): u_{a}\left(A_{a}\right) \geq u_{a}\left(A_{b} \backslash\{o\}\right)$.

An allocation that is $\mathrm{EFX}_{0}$ further satisfies $\mathrm{EFX}$. Also, $\mathrm{EFX}$ is stronger than EF1. It is well-known that the opposite relations might not hold.

Envy-freeness by parts Let $A=\left(A_{1}, \ldots, A_{n}\right)$ be a given allocation. We let $A_{a}^{+}=\left\{o \in A_{a} \mid u_{a}(o)>0\right\}$ and $A_{a}^{-}=\left\{o \in A_{a} \mid u_{a}(o)<0\right\}$ for each $a \in[n]$. Envy-freeness by parts up to one item ensures that EF1 (or EFX) is satisfied in each of the allocations $A, A^{+}=\left(A_{1}^{+}, \ldots, A_{n}^{+}\right)$and $A^{-}=\left(A_{1}^{-}, \ldots, A_{n}^{-}\right)$.

Definition $4\left(\mathrm{EF} 1^{3}\right)$ An allocation $A$ is envy-free by parts up to some item (EF1-EF1-EF1 or $\mathrm{EF}^{3}{ }^{3}$ ) if the following conditions hold: (1) $A$ is EF1, (2) $A^{+}$ is EF1 and (3) $A^{-}$is EF1.

Definition $5\left(\mathrm{EFX}^{3}\right)$ An allocation $A$ is envy-free by parts up to any item (EFX-EFX-EFX or $\mathrm{EFX}^{3}$ ) if the following conditions hold: (1) $A$ is EFX, (2) $A^{+}$is EFX and (3) $A^{-}$is EFX.

With just goods (bads), $\mathrm{EF}^{3}\left(\mathrm{EFX}^{3}\right)$ is $\mathrm{EF} 1$ (EFX). With mixed manna, an allocation that is $\mathrm{EF}^{3}$ also satisfies EF1, one that is $\mathrm{EFX}^{3}$ satisfies EFX, and one that is $\mathrm{EFX}^{3}$ satisfies $\mathrm{EF}^{3}$. The reverse implications might not be true. 
Pareto-optimality We also study each of these fairness properties in combination with an efficiency criterion such as Pareto-optimality (PO), proposed a long time ago by Vilfredo Pareto [24].

Definition 6 (PO) An allocation $A$ is Pareto-optimal if there is no allocation $B$ that Pareto-improves $A$, i.e. $\forall a \in[n]: u_{a}\left(B_{a}\right) \geq u_{a}\left(A_{a}\right)$ and $\exists b \in[n]: u_{b}\left(B_{b}\right)>$ $u_{b}\left(A_{b}\right)$.

\section{Further motivation}

We next further motivate the new properties $\mathrm{EF}^{3}$ and $\mathrm{EFX}^{3}$ by means of a simple example. Consider a birthday party where Bob invites his new friends Alice and Mary. Bob has 3 pieces of his favourite strawberry cake (value is 1) and 2 pieces of the less favorable to him chocolate cake (value is 0). Bob also hopes that some of his guests would be willing to help him washing up the dishes and throwing away the garbage after the party. Alice and Mary arrive and it turns out that both like only chocolate cake (value is 1), and dislike any of the household chores (value is -1 ) as does Bob. How shall we allocate the 5 goods (i.e. all pieces of cake) and the 2 chores?

For EF1 (EFX) and PO, we shall give the strawberry cake to Bob and one piece of the chocolate cake to each of Alice and Mary. As a result, Bob gets utility 3 whereas Alice and Mary get each utility 1 . If we want to maximize the egalitarian welfare, we should assign both chores to Bob. Doing so preserves EF1 (EFX) and PO for all items. However, it violates $\mathrm{EF}^{3}\left(\mathrm{EFX}^{3}\right)$. Indeed,

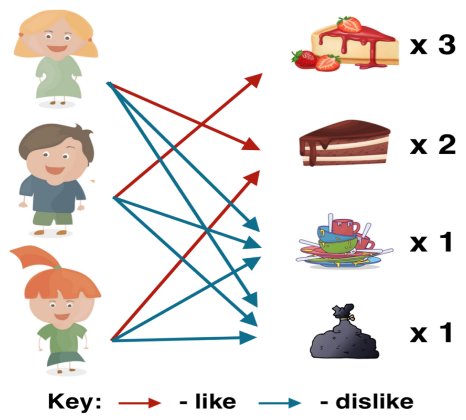
Bob might be unhappy simply because they have to do both chores instead of sharing them with Alice and Mary. This means that an EF1 (EFX) allocation might not satisfy $\mathrm{EF}^{3}\left(\mathrm{EFX}^{3}\right)$. In contrast, achieving $\mathrm{EF}^{3}\left(\mathrm{EFX}^{3}\right)$ avoids assigning both chores to Bob. For example, asking Bob to wash up the dishes and Alice to throw away the garbage, or vice versa is $\mathrm{EF}^{3}{ }^{3}\left(\mathrm{EFX}^{3}\right)$. Other such options share the chores between Bob and Mary, and Alice and Mary. However, none of these maximizes the egalitarian welfare. This means that $\mathrm{EF}^{1}{ }^{1}\left(\mathrm{EFX}^{3}\right)$ is incompatible with this objective.

\section{$5 \quad$ General additive utilities}

We begin with general utilities. An EF1 allocation in this domain can be computed in $O\left(\max \left\{m^{2}, m n\right\}\right)$ time. For this purpose, we can use the existing double round-robin algorithm from [4]. However, this algorithm may fail to guarantee PO because an agent might pick a bad for which some other agent have zero utility. 
Example 1 Consider 2 agents and 2 items, say a and $b$. Define the utilities as follows: $u_{1}(a)=-1, u_{1}(b)=-1$ and $u_{2}(a)=-1, u_{2}(b)=0$. In this problem, the double round-robin algorithm is simply a round-robin rule with some strict priority ordering of the agents. Wlog, let agent 1 pick before agent 2. Wlog, let agent 1 pick $b$. Now, agent 2 can only pick a. The returned allocation gives utility -1 to agent 1 and utility -1 to agent 2. By swapping these items, agent 1 receive utility -1 and agent 2 receive utility 0 . Clearly, this is a Pareto-improvement.

In response, we modify slightly the double round-robin algorithm by adding an extra preliminary phase where each dummy item/non-pure bad is allocated to an agent who has zero utility for it: Algorithm 1 . As we show, this modified version gives us an $\mathrm{EF}^{3}$ allocation that is $\mathrm{PO}$ not only with $-1 / 0 / 1$ utilities but also with any ternary utilities, as well as with absolute identical utilities.

Theorem 1 With general utilities, Algorithm 1 returns an $E F 1^{3}$ allocation in $O\left(\max \left\{m^{2}, m n\right\}\right)$ time.

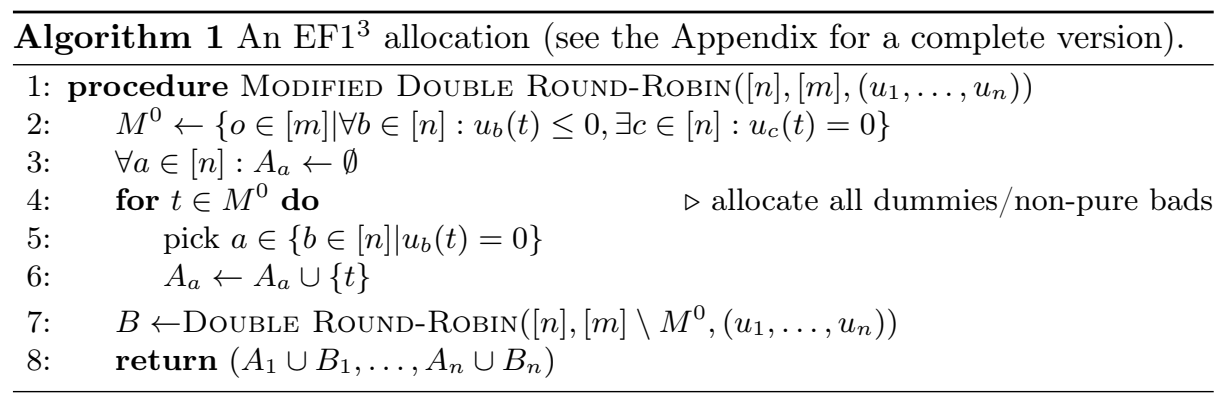

Proof. The double round-robin algorithm returns an EF1 allocation, and so $B$ is EF1. Consider $B^{+}$and $B^{-}$. Let there be $q n-p$ pure bads for some $q, p \in \mathbb{N}$ with $p<n$. The algorithm creates $p$ "fake" dummy items for which each agent has utility 0 , and adds them to the set of pure bads. Hence, the number of items in this set becomes $q n$. Thus, the agents come in a round-robin fashion according to some ordering of the agents, say $(1, \ldots, n-1, n)$, and pick their most preferred item in this set (i.e. all pure bads and "fake" dummies) until all of them are allocated. This is EF1 for the pure bads. Hence, $B^{-}$is EF1.

Further, the agents come in a round-robin fashion by following the reversed ordering, i.e. $(n, n-1, \ldots, 1)$, and pick their most preferred good until all mixed items and goods are allocated. If an agent has no available item which gives them strictly positive utility, they pretend to pick a new "fake" dummy item for which they have utility 0 . This is EF1 for the mixed items and goods. Hence, $B^{+}$is also EF1 which implies that $B$ is $E F 1^{3}$. Finally, extending $B$ to all items, by allocating each dummy item/non-pure bad to someone who holds zero utility, preserves $\mathrm{EF}^{3}{ }^{3}$. This means that the returned allocation is $\mathrm{EF} 1^{3}$. 
We move to stronger properties. For example, $\mathrm{EFX}^{3}$ allocations in our setting might not exist. The rationale behind this is that an agent may get their least valued bad in an attempt of achieving EFX for the bads. As a result, removing this bad from their bundle might not be sufficient to eliminate their envy of some other agent who receive positive utility for a good and a bad.

Proposition 1 There are problems with 2 agents and ternary identical utilities for 1 pure goods and 2 pure bads, in which no allocation is EF $X^{3}$.

Proof. Suppose that there are 2 agents and 3 items. We define the utilities as follows: $u(a)=-1, u(b)=-1, u(c)=2$. We note that one EFX allocation gives items $a, b$ and $c$ to agent 1 and no items to agent 2. However, there is no allocation that satisfies $\mathrm{EFX}^{3}$.

We observe that there are two EFX allocations of the pure bads, i.e. $A=$ $(\{a\},\{b\})$ and $B=(\{b\},\{a\})$. Further, we observe that there are two EFX allocations of the pure good, i.e. $C=(\{c\}, \emptyset)$ and $D=(\emptyset,\{c\})$. By the symmetry of the utilities, we consider only $A, C$ and $D$.

If we unite ("agent-wise") $A$ and $C$, then $u\left(A_{2} \cup C_{2} \backslash\{b\}\right)=0<1=u\left(A_{1} \cup C_{1}\right)$. Therefore, the union of $A$ and $C$ is not EFX and, therefore, $\mathrm{EFX}^{3}$. If we unite $A$ and $D$, then $u\left(A_{1} \cup D_{1} \backslash\{a\}\right)=0<1=u\left(A_{2} \cup D_{2}\right)$. Again, the union of $A$ and $D$ violates $\mathrm{EFX}^{3}$. Similarly, for $B, C$ and $D$.

By comparison, EFX allocations exist in 2-value problems with goods [2]. It follows immediately that $\mathrm{EFX}^{3}$ allocations exist in such problems. From this perspective, we feel that our impossibility result compares favorably to this possibility result because such allocations may not exist in 2-value problems with goods and bads.

Even more, this result also implies that no EFX allocation satisfies $\mathrm{EF} 1^{3}$ and no $\mathrm{EF}^{3}$ allocation satisfies EFX in some problems with identical and ternary utilities. As a consequence, any allocation that could be returned by Algorithm 1 might violate EFX. These implications are also true for the stronger version $\mathrm{EFX}_{0}$ in problems where such allocations exist.

However, $\mathrm{EFX}_{0}$ allocations might also not always exist. The reason for this might be the presence of dummies. One may argue that such items could be removed. However, some web-applications on Spliddit for example ask agents to announce items (e.g. inherited items) and utilities but the system has no access to the actual items and, therefore, cannot remove the dummies [15].

Proposition 2 There are problems with 2 agents and ternary identical utilities for 1 pure good and 1 dummy, in which no allocation is $E F X_{0}$.

Proof. Suppose that there are 2 agents and 2 items, say $a$ and $b$. We define the utilities as follows: $u(a)=1$ and $u(b)=0$. We argue that there is no $\mathrm{EFX}_{0}$ allocation in this problem. To see this, we make two observations. Firstly, with the given set of items, it is impossible that both agents obtain the same utility, as the individual utilities are integers and their sum is odd. Secondly, $\mathrm{EFX}_{0}$ for the agents in this problem where a dummy item is present requires that both agents have the same utility. This follows by the definition of $\mathrm{EFX}_{0}$. 
This result is perhaps interesting because $\mathrm{EFX}_{0}$ allocations exist in problems with 2 agents and general utilities for goods [25], or any number of agents and $0 / 1$ utilities [2]. By Propositions 1 and 2 , it follows that neither $\mathrm{EFX}^{3}$ nor $\mathrm{EFX}_{0}$ can be achieved in combination with $\mathrm{PO}$, or even a weaker efficiency notion such as completeness (i.e. all items are allocated), in general.

\section{Absolute identical additive utilities}

We continue with absolute identical utilities. Requiring such utilities is not as strong as requiring just identical utilities. To see this, consider agents 1, 2 and items $a, b$. Define the utilities as $u_{1}(a)=3, u_{1}(b)=2$ and $u_{2}(a)=3, u_{2}(b)=-2$. The absolute values of these utilities are identical but their cardinal values are not, e.g. $\left|u_{1}(b)\right|=\left|u_{2}(b)\right|=2$ but $u_{1}(b)=2, u_{2}(b)=-2$.

By Proposition 1, EF $1^{3}$ and EFX are incompatible in this domain. Nevertheless, we can combine each of them with PO. For example, Algorithm 1 returns an allocation that satisfies $\mathrm{PO}$ besides $\mathrm{EF}^{3}$. The key reason for this result is that in such problems there are no items that are bads (goods) for some agents and dummy for other agents.

Theorem 2 With absolute identical utilities, Algorithm 1 returns an $E F 1^{3}$ and PO allocation.

Proof. EF $1^{3}$ follows by Theorem 1. We note that each allocation that gives at least one mixed item to an agent who values it strictly negatively can be Pareto-improved by moving this item to an agent who values it strictly positively. Therefore, such an allocation is not Pareto-optimal. We also note that each other allocation, including the returned one, maximizes the sum of agents' utilities because it achieves the maximum utility for each individual item. Such an allocation is always Pareto-optimal.

At the same time, we can compute an EFX and PO allocation in polynomial time. For this task, we propose a new algorithm: Algorithm 2. We let $M(o)=$ $\max _{a \in[n]} u_{a}(o)$ denote the maximum utility that an agent derives from item $o$. Further, let us arrange the items in non-increasing absolute maximum utility order by using the following tie-breaking rule.

Ordering $\sigma_{m}$ : Wlog, $|M(1)| \geq \ldots \geq|M(m)|$. Initialize $\sigma_{m}$ to $(1, \ldots, m)$. While there are two items $s$ and $t$ from $[m]$ such that $|M(s)|=|M(t)|, M(s)>0$, $M(t)<0$ and $t$ is right before $s$ in $\sigma_{m}$, do move $s$ right before $t$ in $\sigma_{m}$. Thus, within items with the same maximum absolute utility, $\sigma_{m}$ gives higher priority to the mixed items/goods than to the pure bads.

Algorithm 2 allocates the items one-by-one in such an ordering $\sigma_{m}$. If the current item $t$ is mixed or pure good, then Algorithm 2 gives it to an agent who has currently the minimum utility among the agents who like the item. If item $t$ is pure bad, then Algorithm 2 gives it to an agent who has currently the maximum utility. Otherwise, it gives item $t$ to an agent with zero utility.

Theorem 3 With absolute identical utilities, Algorithm 2 returns an EFX and $P O$ allocation in $O(\max \{m \log m, m n\})$ time. 


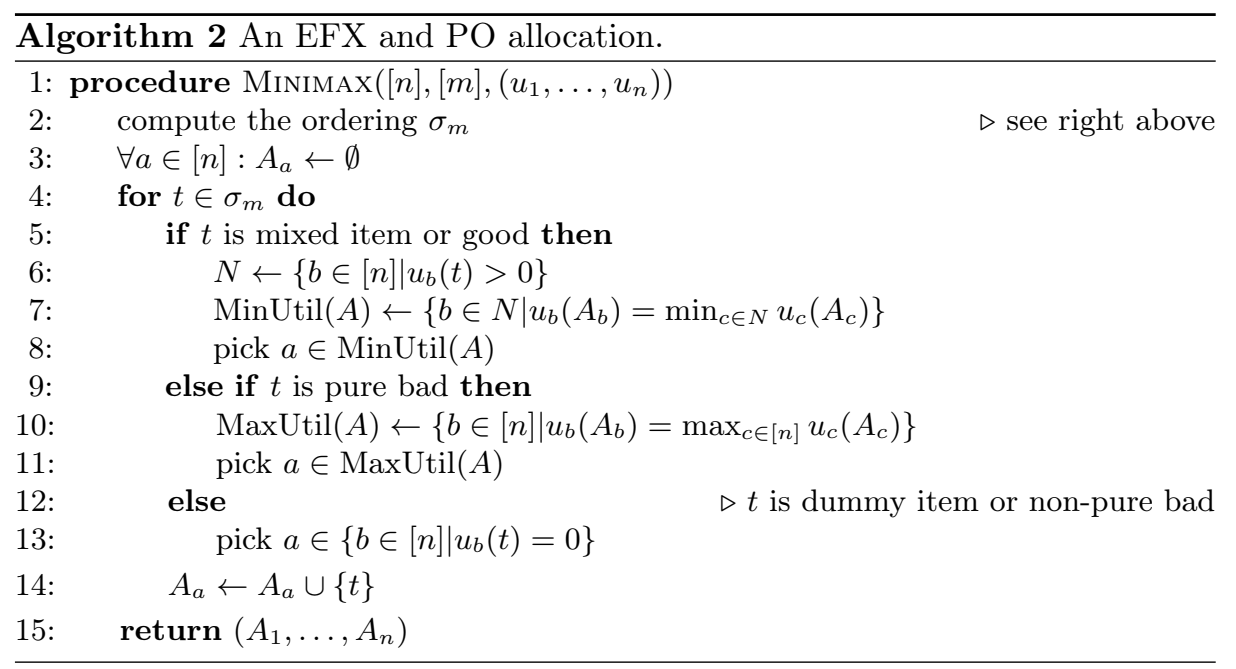

Proof. For $t \in[m]$, we let $A^{t}$ denote the partially constructed allocation of items 1 to $t$. Pareto-optimality of $A^{t}$ follows by the same arguments as in Theorem 2, but now applied to the sub-problem of the first $t$ items. As a result, $A^{m}$ (i.e. the returned allocation) satisfies also PO.

We next prove that $A^{t}$ is EFX by induction on $t \in[m]$. This will imply the result for EFX of $A^{m}$ (i.e. the returned allocation). In the base case, let $t$ be 1 . The allocation of item 1 is trivially EFX. In the hypothesis, let $t>1$ and assume that the allocation $A^{t-1}$ is EFX. In the step case, let us consider round $t$.

Wlog, let the algorithm give item $t$ to agent 1 . That is, $A_{1}^{t}=A_{1}^{t-1} \cup\{t\}$ and $A_{a}^{t}=A_{a}^{t-1}$ for each $a \in[n] \backslash\{1\}$. It follows immediately by the hypothesis that each pair of different agents from $[n] \backslash\{1\}$ is EFX of each other in $A^{t}$. We note that $t$ gives positive, negative or zero utility to agent 1 . For this reason, we consider three remaining cases for agent $a \in[n] \backslash\{1\}$ and agent 1 .

Case 1: Let $u_{1}(t)>0$. In this case, $t$ is mixed item or pure good (good) and $u_{1}\left(A_{1}^{t}\right)>u_{1}\left(A_{1}^{t-1}\right)$ holds. Hence, agent 1 remain EFX of agent $a$ by the hypothesis. For this reason, we next show that agent $a$ is EFX of agent 1 . We consider two sub-cases depending on whether agent $a$ belong to $N=\{b \in$ $\left.[n] \mid u_{b}(t)>0\right\}$ or not. We note that $1 \in N$ holds because of $u_{1}(t)>0$.

Sub-case 1 for $a \rightarrow 1$ : Let $a \notin N$. Hence, $u_{a}(t) \leq 0$. As a result, $u_{a}\left(A_{1}^{t-1}\right) \geq$ $u_{a}\left(A_{1}^{t}\right)$ holds. Thus, as $A^{t-1}$ is EFX, we derive that $u_{a}\left(A_{a}^{t}\right)=u_{a}\left(A_{a}^{t-1}\right) \geq$ $u_{a}\left(A_{1}^{t-1} \backslash\{o\}\right) \geq u_{a}\left(A_{1}^{t} \backslash\{o\}\right)$ holds for each $o \in A_{1}^{t-1}$ with $u_{a}(o)>0$. We also derive $u_{a}\left(A_{a}^{t} \backslash\{o\}\right)=u_{a}\left(A_{a}^{t-1} \backslash\{o\}\right) \geq u_{a}\left(A_{1}^{t-1}\right) \geq u_{a}\left(A_{1}^{t}\right)$ for each $o \in A_{a}^{t}$ with $u_{a}(o)<0$. Hence, agent $a$ is EFX of agent 1 .

Sub-case 2 for $a \rightarrow 1$ : Let $a \in N$. Hence, $u_{a}(t)>0$. Moreover, $u_{a}\left(A_{a}^{t-1}\right) \geq$ $u_{1}\left(A_{1}^{t-1}\right)$ by the selection rule of the algorithm. For each item $o \in A_{1}^{t-1}$, we have $u_{1}(o)=u_{a}(o)$ if $o$ is pure good, pure bad or dummy item, and $u_{1}(o) \geq u_{a}(o)$ if $o$ is mixed item. Therefore, $u_{1}\left(A_{1}^{t-1}\right) \geq u_{a}\left(A_{1}^{t-1}\right)$ or agent $a$ is envy-free of agent 1 in $A^{t-1}$. 
We derive $u_{a}\left(A_{a}^{t}\right)=u_{a}\left(A_{a}^{t-1}\right) \geq u_{a}\left(A_{1}^{t-1}\right)=u_{a}\left(A_{1}^{t} \backslash\{t\}\right)$ because $A_{a}^{t}=A_{a}^{t-1}$ and $A_{1}^{t}=A_{1}^{t-1} \cup\{t\}$. Furthermore, $u_{a}\left(A_{1}^{t} \backslash\{t\}\right) \geq u_{a}\left(A_{1}^{t} \backslash\{o\}\right)$ for each $o \in A_{1}^{t-1}$ with $u_{a}(o)>0$ because $u_{a}(o) \geq u_{a}(t)$ holds due to the ordering of items used by the algorithm.

We now show EFX of the bads. We have $u_{a}\left(A_{a}^{t} \backslash\{o\}\right)=u_{a}\left(A_{a}^{t-1} \backslash\{o\}\right) \geq$ $u_{a}\left(A_{1}^{t-1}\right)+u_{a}(t)=u_{a}\left(A_{1}^{t}\right)$ for each $o \in A_{a}^{t-1}$ with $u_{a}(o)<0$ because $\left|u_{a}(o)\right| \geq$ $u_{a}(t)$ holds due to the ordering of items used by the algorithm. Hence, agent $a$ is EFX of agent 1.

Case 2: Let $u_{1}(t)<0$. In this case, $t$ is pure bad and $u_{a}\left(A_{1}^{t}\right)<u_{a}\left(A_{1}^{t-1}\right)$ holds. That is, agent 1's utility decreases. By the hypothesis, it follows that agent $a$ remain EFX of agent 1 in $A^{t}$. For this reason, we only show that agent 1 remain EFX of agent $a$.

$1 \rightarrow a$ : We have $u_{1}\left(A_{1}^{t-1}\right) \geq u_{a}\left(A_{a}^{t-1}\right)$ by the selection rule of the algorithm. For each item $o \in A_{a}^{t-1}$, we have $u_{a}(o)=u_{1}(o)$ if $o$ is pure good, pure bad or dummy item, and $u_{a}(o) \geq u_{1}(o)$ if $o$ is mixed item. We conclude $u_{a}\left(A_{a}^{t-1}\right) \geq$ $u_{1}\left(A_{a}^{t-1}\right)$ and, therefore, $u_{1}\left(A_{1}^{t-1}\right) \geq u_{1}\left(A_{a}^{t-1}\right)$. Hence, agent 1 is envy-free of agent $a$ in $A^{t-1}$.

Additionally, it follows that $u_{1}\left(A_{1}^{t} \backslash\{t\}\right) \geq u_{1}\left(A_{a}^{t}\right)$ holds because $A_{1}^{t} \backslash\{t\}=$ $A_{1}^{t-1}$ and $A_{a}^{t}=A_{a}^{t-1}$. Due to the order of the items, we have $\left|u_{1}(b)\right| \geq\left|u_{1}(t)\right|$ for each $b \in A_{1}^{t}$ with $u_{1}(b)<0$. Hence, $u_{1}\left(A_{1}^{t} \backslash\{b\}\right) \geq u_{1}\left(A_{1}^{t} \backslash\{t\}\right) \geq u_{1}\left(A_{a}^{t}\right)$ for each $b \in A_{1}^{t}$ with $u_{1}(b)<0$.

At the same time, $u_{1}\left(A_{1}^{t-1}\right) \geq u_{1}\left(A_{a}^{t-1} \backslash\{g\}\right)$ for each $g \in A_{a}^{t-1}$ with $u_{1}(g)>$ 0 . Again, due to the order of the items, $u_{1}(g) \geq\left|u_{1}(t)\right|$. Therefore, $u_{1}\left(A_{a}^{t}\right\rangle$ $\{g\}) \leq u_{1}\left(A_{a}^{t}\right)-\left|u_{1}(t)\right|=u_{1}\left(A_{a}^{t-1}\right)-\left|u_{1}(t)\right| \leq u_{1}\left(A_{1}^{t-1}\right)-\left|u_{1}(t)\right|=u_{1}\left(A_{1}^{t}\right)$. Consequently, $u_{1}\left(A_{1}^{t}\right) \geq u_{1}\left(A_{a}^{t} \backslash\{g\}\right)$ for each $g \in A_{a}^{t}$ with $u_{1}(g)>0$.

Case 3: Let $u_{1}(t)=0$. In this case, $t$ is dummy item or non-pure bad. Hence, $u_{a}\left(A_{1}^{t-1}\right) \geq u_{a}\left(A_{1}^{t}\right)$ and $u_{1}\left(A_{1}^{t}\right)=u_{1}\left(A_{1}^{t-1}\right)$ hold. That is, agent 1's utility does not change. By the hypothesis, this means that they remain EFX of each agent $a$ and also each agent $a$ remains EFX of them in $A^{t}$.

Finally, computing maximum values takes $O(m n)$ time and sorting items takes $O(m \log m)$ time. The loop of the algorithm takes $O(m n)$ time.

For problems with identical utilities, Aziz and Ray [6] proposed the "egalsequential" algorithm for computing EFX and PO allocations. By Theorem 3 , Algorithm 2 also does that. However, we feel that such problems are very restrictive as they do not have mixed items unlike many practical problems.

Corollary 1 With identical utilities, Algorithm 2 returns an EFX and PO allocation.

Algorithm/2allocates each mixed item/good to an agent who likes it, and each dummy item/non-pure bad to an agent who is indifferent to it. As a consequence, the result in Theorem 3 extends to problems where, for each mixed item/good, the likes are identical and, for each pure bad, the dislikes are identical.

Corollary 2 With identical likes (i.e. strictly positive utilities) for each mixed item, identical likes for each good and identical dislikes (i.e. strictly negative utilities) for each pure bad, Algorithm 2 returns an EFX and PO allocation. 


\section{$7 \quad$ Ternary additive utilities}

We end with ternary utilities. That is, each agent's utility for each item is from $\{-\alpha, 0, \beta\}$ where $\alpha, \beta \in \mathbb{R}_{>0}$. We consider two cases for such utilities.

\subsection{Case for any $\alpha, \beta$}

By Proposition 1, it follows that an $\mathrm{EFX}^{3}$ allocation might not exist in some problems even when $\alpha=1$ and $\beta=2$. However, we can compute an EF1 ${ }^{3}$ (notably, also EF1-EFX-EFX) and PO allocation with Algorithm 1 .

Theorem 4 With ternary utilities from $\{-\alpha, 0, \beta\}$ where $\alpha, \beta \in \mathbb{R}_{>0}$, Algorithm 1 returns an $E F 1^{3}$ and $P O$ allocation.

Proof. The returned allocation is $\mathrm{EF} 1^{3}$ by Theorem 1 1 . This one achieves the maximum utility for each individual item. Hence, the sum of agents' utilities in it is maximized and equal to $\beta$ multiplied by the number of goods plus $\beta$ multiplied by the number of mixed items minus $\alpha$ multiplied by the number of pure bads. In fact, this holds for each allocation that gives each mixed item/good to an agent who has utility $\beta$, and each dummy/non-pure bad to an agent who has utility 0 . Each other allocation is not PO and does not Pareto-dominate the returned allocation. Hence, the returned one is PO.

One the other hand, we already mentioned after Proposition 1 that each allocation returned by Algorithm 1 in such problems may violate EFX. However, Algorithm 2 returns an EFX and PO allocation in this case.

Theorem 5 With ternary utilities from $\{-\alpha, 0, \beta\}$ where $\alpha, \beta \in \mathbb{R}_{>0}$, Algorithm 0 returns an EFX and PO allocation.

Proof. This is where the ordering used by the algorithm plays a crucial role. If $\beta \geq \alpha$, we note that all mixed items and goods are allocated before all pure bads and all of these are allocated before the remaining items (i.e. dummy items and non-pure bads). If $\beta<\alpha$, we note that all pure bads are allocated before all mixed items and goods and all of these are allocated before the remaining items. Further, we observe that agents have identical likes for each mixed item or each good (i.e. $\beta$ ), and identical dislikes for each pure bad (i.e. $-\alpha$ ). Therefore, the result follows by Corollary 2 .

By comparison, the "ternary flow" algorithm of Aziz and Rey [6] may fail to return an EFX allocation even with $-2 / 1$ utilities. To see this, simply negate the utilities in the problem from Proposition 1 . This algorithm allocates firstly one good to each agent and secondly the bad to one of the agents. This outcome violates EFX.

\subsection{Case for $\alpha=\beta$}

In this case, we can compute an $\mathrm{EFX}^{3}$ and $\mathrm{PO}$ allocation with Algorithm 1 . Although we consider this a minor result, we find it important because it is the only one in our analysis when $\mathrm{EFX}^{3}$ and $\mathrm{PO}$ allocations exist. 
Theorem 6 With ternary utilities from $\{-\alpha, 0, \alpha\}$ where $\alpha \in \mathbb{R}_{>0}$, Algorithm 1 returns an $E F X^{3}$ and $P O$ allocation.

Proof. The returned allocation is $\mathrm{EF} 1^{1}$ and $\mathrm{PO}$ by Theorem 4 . With general (and, therefore, ternary) utilities, an allocation that is EFX ${ }^{3}$ also satisfies EF1 ${ }^{3}$ because EFX is a stronger property than EF1, but the opposite implication might not be true. Well, with utilities from $\{-\alpha, 0, \alpha\}$, the opposite implication also holds. Indeed, if an allocation is EF1 for a given pair of agents, then removing some good from the envied agent's bundle or removing some bad from the envy agent's bundle eliminates the envy of the envy agent. But, the envy agent likes each such good with $\alpha$ and each such bad with $-\alpha$. Hence, such an allocation is EFX. This implies that an $\mathrm{EF}^{3}$ allocation is also $\mathrm{EFX}^{3}$ in this domain.

By Theorem 5. Algorithm 2 returns an EFX and PO allocation in this case. However, this one might falsify $\mathrm{EFX}^{3}$ even when $\alpha=1$ (see motivating example). The same holds for the "ternary flow" algorithm of Aziz and Rey [6] because it maximizes the egalitarian welfare when $\alpha=1$ (see motivating example).

\section{Conclusions}

We considered additive and fair division of mixed manna. For this model, we analysed axiomatic properties of allocations such as $\mathrm{EFX}_{0}, \mathrm{EFX}^{3}, \mathrm{EFX}, \mathrm{EF}^{3}$, EF1 and PO in three utility domains. With general utilities, we showed that an $\mathrm{EF} 1^{3}$ allocation exists and gave Algorithm 1 for computing such an allocation (Theorem 11). With absolute identical or $-\alpha / 0 / \beta$ utilities, this algorithm returns an $\mathrm{EF}^{3}$ and $\mathrm{PO}$ allocation (Theorems 2 and 4 . With $-\alpha / 0 / \alpha$ utilities, it returns an $\mathrm{EFX}^{3}$ and PO allocation (Theorem 6).

With absolute identical utilities, we gave Algorithm 2 for computing an EFX and PO allocation (Theorem 3). With ternary utilities, this algorithm also returns such an allocation (Theorem 5). We further proved two impossibilities results (Propositions 1 and 22. In particular, with ternary identical utilities, an $\mathrm{EFX}_{0}$ allocation, or an $\mathrm{EFX}^{3}$ allocation might not exist. We leave for future work two very interesting open questions with general utilities. Table 1 contains our results.

Table 1. Key: $\checkmark$-possible, $\times$-not possible, P-polynomial time, $\alpha, \beta \in \mathbb{R}_{>0}: \alpha \neq \beta$.

\begin{tabular}{|c|c|c|c|c|}
\hline property & $\begin{array}{l}\text { general } \\
\text { utilities }\end{array}$ & $\begin{array}{c}\text { ident. \& abs. } \\
\text { utilities }\end{array}$ & $\begin{array}{l}-\alpha / 0 / \beta \\
\text { utilities }\end{array}$ & $\begin{array}{l}-\alpha / 0 / \alpha \\
\text { utilities }\end{array}$ \\
\hline $\mathrm{EF}^{3}{ }^{3}$ & \multicolumn{4}{|c|}{$\checkmark, P(\operatorname{Thm} 1)$} \\
\hline $\mathrm{EF}^{3} \& \mathrm{PO}$ & open & $\checkmark$, P (Thm 2) & $\checkmark, P(T h m 4)$ & \\
\hline EFX \& PO & open & $\checkmark, \mathrm{P}(\mathrm{Thm} 3)$ & $\checkmark, \mathrm{P}(\mathrm{Thm} \overline{5})$ & \\
\hline $\begin{array}{c}\mathrm{EFX}^{3} \\
\mathrm{EFX}^{3} \& \mathrm{PO}\end{array}$ & \multicolumn{3}{|c|}{$\times($ Prop 1$)$} & $\checkmark, \mathrm{P}(\mathrm{Thm} 6)$ \\
\hline $\mathrm{EFX}_{0}$ & & $\times($ Prop 2$)$ & & \\
\hline
\end{tabular}




\section{References}

[1] Aleksandrov, M., Aziz, H., Gaspers, S., Walsh, T.: Online fair division: analysing a food bank problem. In: Proceedings of the Twenty-Fourth IJCAI 2015, Buenos Aires, Argentina, July 25-31, 2015. pp. 2540-2546 (2015)

[2] Amanatidis, G., Birmpas, G., Filos-Ratsikas, A., Hollender, A., Voudouris, A.A.: Maximum Nash welfare and other stories about EFX (2020)

[3] Amanatidis, G., Birmpas, G., Markakis, V.: Comparing approximate relaxations of envy-freeness. In: Proceedings of the Twenty-Seventh International Joint Conference on Artificial Intelligence, IJCAI 2018, Stockholm, Sweden, July 13-19, 2018. pp. 42-48 (2018)

[4] Aziz, H., Caragiannis, I., Igarashi, A., Walsh, T.: Fair allocation of indivisible goods and chores. In: Proceedings of the Twenty-Eighth International Joint Conference on Artificial Intelligence, IJCAI-19. pp. 53-59. International Joint Conferences on Artificial Intelligence Organization (7 2019)

[5] Aziz, H., Moulin, H., Sandomirskiy, F.: A polynomial-time algorithm for computing a Pareto optimal and almost proportional allocation. CoRR abs/1909.00740 (2019)

[6] Aziz, H., Rey, S.: Almost group envy-free allocation of indivisible goods and chores. CoRR abs/1907.09279 (2019)

[7] Barman, S., Krishnamurthy, S.K., Vaish, R.: Finding fair and efficient allocations. In: Proceedings of the 2018 ACM Conference on EC 2018. pp. 557-574. ACM, New York, NY, USA (2018)

[8] Barman, S., Krishnamurthy, S.K., Vaish, R.: Greedy algorithms for maximizing Nash social welfare. In: Proceedings of the 17th International Conference on $\mathrm{Au}-$ tonomous Agents and MultiAgent Systems, AAMAS 2018, Stockholm, Sweden, July 10-15, 2018. pp. 7-13 (2018)

[9] Bogomolnaia, A., Moulin, H., Sandomirskiy, F., Yanovskaia, E.: Dividing bads under additive utilities. Social Choice and Welfare 52(3), 395-417 (2019)

[10] Bogomolnaia, A., Moulin, H., Sandomirskiy, F., Yanovskaya, E.: Dividing goods and bads under additive utilities. CoRR abs/1610.03745 (2016)

[11] Bogomolnaia, A., Moulin, H., Sandomirskiy, F., Yanovskaya, E.: Competitive division of a mixed manna. Econometrica 85(6), 1847-1871 (2017)

[12] Brams, S.J., Taylor, A.D.: Fair division - from cake-cutting to dispute resolution. Cambridge University Press (1996)

[13] Budish, E.: The combinatorial assignment problem: Approximate competitive equilibrium from equal incomes. Journal of Political Economy 119(6), 1061-1103 (2011)

[14] Caragiannis, I., Kaklamanis, C., Kanellopoulos, P., Kyropoulou, M.: The efficiency of fair division. Theory of Computing Systems 50(4), 589-610 (May 2012)

[15] Caragiannis, I., Kurokawa, D., Moulin, H., Procaccia, A.D., Shah, N., Wang, J.: The unreasonable fairness of maximum Nash welfare. In: Proceedings of ACM Conference on EC '16, Maastricht, The Netherlands, July 24-28, 2016. pp. 305$322(2016)$

[16] Chevaleyre, Y., Dunne, P., Endriss, U., Lang, J., Lemaitre, M., Maudet, N., Padget, J., Phelps, S., Rodrguez-Aguilar, J., Sousa, P.: Issues in multiagent resource allocation. Informatica 30, 3-31 (2006)

[17] Dobzinski, S., Vondrák, J.: Communication complexity of combinatorial auctions with submodular valuations. In: Proceedings of the Twenty-fourth Annual ACMSIAM Symposium on Discrete Algorithms. pp. 1205-1215. SODA '13, Society for Industrial and Applied Mathematics, Philadelphia, PA, USA (2013) 
[18] Dubins, L.E., Spanier, E.H.: How to cut a cake fairly. The American Mathematical Monthly 68(1P1), 1-17 (1961)

[19] Foley, D.K.: Resource allocation and the public sector. Yale Economic Essays 7(1), 45-98 (1967)

[20] Hugo, S.: The problem of fair division. Econometrica 16, 101-104 (1948)

[21] Kyropoulou, M., Suksompong, W., Voudouris, A.: Almost envy-freeness in group resource allocation. In: Proceedings of the Twenty-Eighth International Joint Conference on Artificial Intelligence, IJCAI-19. pp. 400-406. International Joint Conferences on Artificial Intelligence Organization (08 2019)

[22] Lipton, R.J., Markakis, E., Mossel, E., Saberi, A.: On approximately fair allocations of indivisible goods. In: Proceedings of the 5th ACM Conference on EC, New York, NY, USA, May 17-20, 2004. pp. 125-131 (2004)

[23] Moulin, H.: Fair division and collective welfare. MIT Press (2003)

[24] Pareto, V.: Cours d'Économie politique. Professeur á l'Université de Lausanne. Vol. I. Pp. 430. 1896. Vol. II. Pp. 426. 1897. Lausanne: F. Rouge (1897)

[25] Plaut, B., Roughgarden, T.: Almost envy-freeness with general valuations. In: Proceedings of the 29th Annual ACM-SIAM Symposium on Discrete Algorithms, SODA 2018, New Orleans, LA, USA, January 7-10, 2018. pp. 2584-2603 (2018)

[26] Sandomirskiy, F., Segal-Halevi, E.: Fair division with minimal sharing. CoRR abs/1908.01669 (2019)

[27] Young, H.P.: Equity - in theory and practice. Princeton University Press (1995)

\section{A A complete version of Algorithm 1}

For reasons of space, we presented a short version of Algorithm 1 in the main text. We present in here a complete version of it.

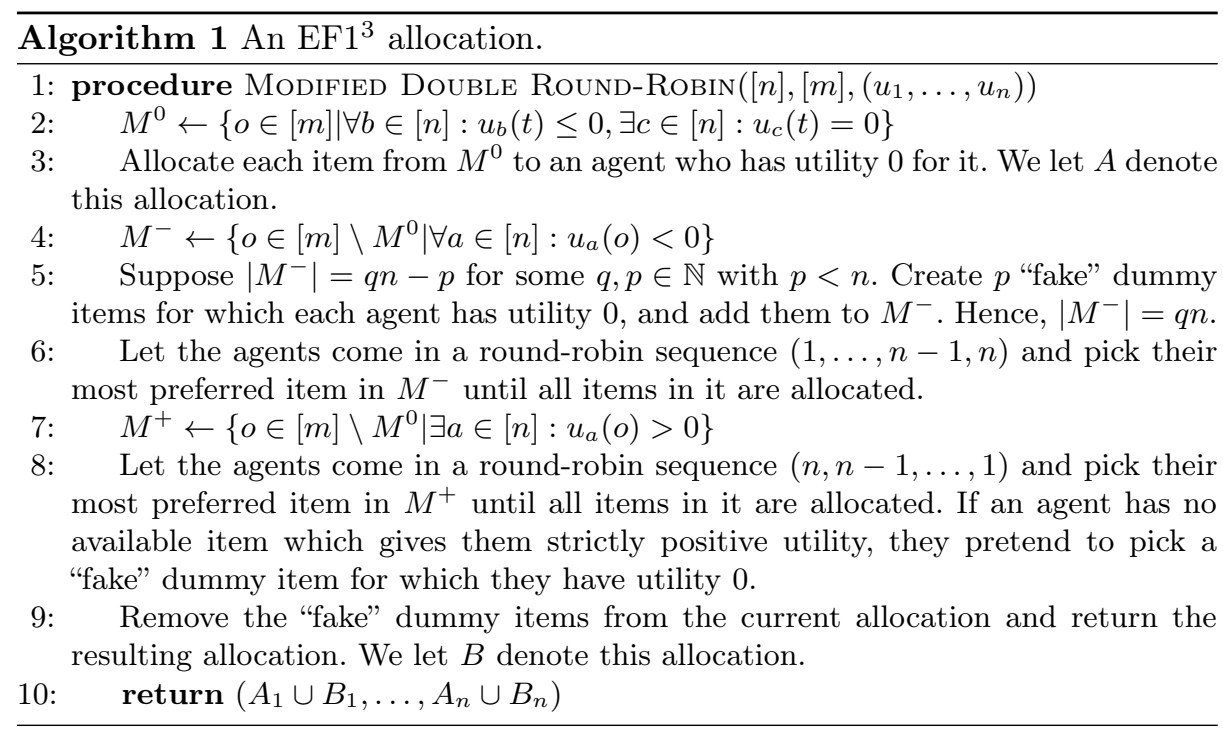

